\section{ЯДЕРНА ФІЗИКА} NUCLEAR PHYSICS

УДК 539.166+539.17

https://doi.org/10.15407/jnpae2019.03.213

\author{
V. A. Plujko ${ }^{1,2}$, S. Goriely ${ }^{3}$, O. M. Gorbachenko ${ }^{1}$, K. M. Solodovnyk ${ }^{1, *}$ \\ ${ }^{I}$ Nuclear Physics Department, Taras Shevchenko National University of Kyiv, Kyiv, Ukraine \\ ${ }^{2}$ Institute for Nuclear Research, National Academy of Sciences of Ukraine, Kyiv, Ukraine \\ ${ }^{3}$ Institut d'Astronomie et d'Astrophysique, Université Libre de Bruxelles, Brussels, Belgium
}

*Corresponding author: e.solodovnyk@gmail.com

\title{
TEST OF MODELS FOR PHOTON STRENGTH FUNCTIONS OF ELECTRIC DIPOLE PHOTOEXCITATION
}

Closed-form models for photon strength functions for the description of photoabsorption cross section in the energy range $5-30 \mathrm{MeV}$ for even-even nuclei are tested. The experimental database was prepared with systematic uncertainty less than $10 \%$ from the EXFOR data. The uncertainties are estimated using the calculations of the photo cross sections within TALYS 1.6 code. The theoretical models are compared with experimental photoabsorption cross section data. The minimum of the least-square deviation and the root-mean-square deviation factor are used as a criteria comparison of the theoretical calculations with experimental data. It is shown that the simple modified Lorentzian model is the best approach for simulation of the photoabsorption cross section at the gamma-ray energies below $\sim 30 \mathrm{MeV}$.

Keywords: models of photon strength functions, photoabsorption, giant dipole resonance.

\section{Introduction}

The photonuclear data are being intensively studied in response to growing needs for these data in a variety of practical applications [1]. Photons are commonly produced as bremsstrahlung radiation by electron accelerators which are relatively simple machines present in many hospitals, industries and laboratories. These institutions deal with applications such as activation analyses, radiation shielding and radiation transport analyses, safeguards and inspection technologies (identification of materials through radiation induced by photonuclear reactions using portable bremsstrahlung devices), calculation of absorbed dose in the human body during radiotherapy etc., see [1] for the references. Photon strength functions (PSFs) are important input parameter for modelling nuclear reactions, for the calculations of gamma-ray capture cross sections, gamma-ray production spectra, competition between gamma-ray and particle emission and of various properties of the atomic nuclei (see, for example, [2, 3 ] for references). The PSFs are mandatory component of all modern computer codes for nuclear reaction calculations and nuclear data evaluation, such as EMPIRE and TALYS $[4,5]$.

The electric dipole (E1) multipolarity of gammarays is dominant in bremsstrahlung spectra and dominant in nuclear processes of photoabsorption and gamma-decay when they occur simultaneously with transitions of other multipolarities. For mediumheavy and heavy atomic nuclei, the most important contribution to the probability of these transitions in the range of gamma-ray energies $8<\varepsilon_{\gamma}<30 \mathrm{MeV}$ is resulted from the response of Isovector Giant Dipole Resonances (GDR). A Lorentz shape is preferable for approximation of a PSF of such response (see [2, $3,6,7]$ and references therein). Here we consider the common-used analytical models with this lineshape, namely Standard Lorentzian (SLO) [2, 3], Generalized Lorentzian (GLO) [2, 3, 8], Simplified version of modified Lorentzian (SMLO) [3 - 7, 9 13] and Triple Lorentzian (TLO) $[14,15]$.

The total photoabsorption cross section $\sigma_{\text {abs }}\left(\varepsilon_{\gamma}\right)$ is calculated as a sum of the terms corresponding to the E1 GDR excitation $\sigma_{\mathrm{GDR}}$ and quasi-deuteron photodisintegration $\sigma_{\mathrm{qd}}\left(\varepsilon_{\gamma}\right)$ :

$$
\sigma_{\mathrm{abs}}\left(\varepsilon_{\gamma}\right)=\sigma_{\mathrm{GDR}}\left(\varepsilon_{\gamma}\right)+\sigma_{\mathrm{qd}}\left(\varepsilon_{\gamma}\right) \text {. }
$$

The approach from Ref. $[2,16]$ is used for a quasi-deuteron contribution. The main part of experimental data on photoabsorption cross sections is obtained with the use of bremsstrahlung, which is mainly composed of gamma-rays of E1 multipolarity. Thus, the GDR component of the total photoabsorption cross section is adopted to be equal to the photoabsorption cross section of electric dipole gamma-rays $\sigma_{\mathrm{GDR}}\left(\varepsilon_{\gamma}\right)=\sigma_{\mathrm{E} 1}\left(\varepsilon_{\gamma}\right)$. The average photoabsorption cross section of a nucleus in the ground state of spin $J_{0}$ for excitation of levels of spin $J$ is proportional to the photoexcitation E1 PSF and is given by [6] $\sigma_{\mathrm{E} 1}^{J}=\frac{g_{J}}{3} \sigma_{\mathrm{E} 1}=g_{J}(\pi \hbar c)^{2} \varepsilon_{\gamma} \vec{f}_{\mathrm{E} 1}\left(\varepsilon_{\gamma}\right)$, where $g_{J}=(2 J+1) /\left(2 J_{0}+1\right)$ is the statistical factor and $\left|J_{0}-1\right| \leq J \leq J_{0}+1$. The analytical model 
for E1 PSF of photoexcitation $\vec{f}^{\alpha}\left(\varepsilon_{\gamma}\right)$ defines the component of photoabsorption cross section $\sigma_{\mathrm{GDR}}$ (1) resulted from the GDR excitation:

$$
\sigma_{\mathrm{GD} R}\left(\varepsilon_{\gamma}\right)=\sigma_{\mathrm{GDR}}^{\alpha}\left(\varepsilon_{\gamma}\right)=3(\pi \hbar c)^{2} \varepsilon_{\gamma} \vec{f}^{\alpha}\left(\varepsilon_{\gamma}\right),
$$

where index $\alpha$ denotes the PSF model. General analytical expression for the E1 PSF of photoexcitation of the cold nuclei governed by the GDR can be presented in the following form

$$
\begin{gathered}
\vec{f}^{\alpha}\left(\varepsilon_{\gamma}\right)=8.674 \cdot 10^{-8} \sum_{j=1}^{j_{m}} \frac{\sigma_{\mathrm{TRK}} s_{j}^{\alpha}}{\varepsilon_{\gamma}} \times \\
\times \frac{2}{\pi} \frac{\varepsilon_{\gamma}^{2} \Gamma_{j}^{\alpha}}{\left(\varepsilon_{\gamma}^{2}-\left(E_{r, j}^{\alpha}\right)^{2}\right)^{2}+\left(\Gamma_{j}^{\alpha} \varepsilon_{\gamma}\right)^{2}}\left(\mathrm{MeV}^{-3}\right) .
\end{gathered}
$$

Here, the index $j$ specifies number of the normal modes of giant vibrations: for spherical nuclei $j_{m}=1$, for axially symmetric $j_{m}=2$ and for nuclei with triaxial shape $j_{m}=3$; factor $s_{j}^{\alpha}$ is a weight of the $j$-mode; $\sigma_{\mathrm{TRK}}$ is the Thomas - Reiche - Kuhn (TRK) sum rule $\sigma_{\mathrm{TRK}}=15 A\left(1-I^{2}\right)(\mathrm{mb} \cdot \mathrm{MeV})$, with $I=(N-Z) / A$ for the neutron-proton asymmetry factor. A weight of the $j$-mode determines cross section of $j$-mode at resonance energy $E_{r, j}^{\alpha}$ : $\sigma_{r, j}^{\alpha}=(2 / \pi) \sigma_{\mathrm{TRK}} \cdot s_{j}^{\alpha} / \Gamma_{j}^{\alpha}$.

An approximation of axially symmetric nuclei with the effective quadrupole deformation parameter $\beta_{2}$ is adopted for deformed nuclei in the SLO, GLO and SMLO models. The values of effective quadrupole deformation parameters are taken from "deflib.dat" file of RIPL $2\left(\beta_{2}=\beta_{2, \text { eff }}\right)$ [17]. These models for cold nuclei employing the same general expression (3) but with different determination of the shape width $\Gamma_{j}^{\alpha}$. For the SLO model, the width is energy-independent constant which is equal to GDR width for $j$-mode: $\Gamma_{j}^{\mathrm{SLO}}=\Gamma_{r, j}^{\mathrm{SLO}}$. The width in the GLOmodel is quadratic in gamma-ray energy $\Gamma_{j}^{\mathrm{GLO}}\left(\varepsilon_{\gamma}\right)=\varepsilon_{\gamma}^{2} \cdot \Gamma_{r, j}^{\mathrm{SLO}} /\left(E_{r, j}^{\mathrm{SLO}}\right)^{2}$, and the width in the
SMLO model is a linear function of the gamma-ray energy $\Gamma_{j}^{\mathrm{SMLO}}\left(\varepsilon_{\gamma}\right)=\varepsilon_{\gamma} \cdot \Gamma_{r, j}^{\mathrm{SMLO}} / E_{r, j}^{\mathrm{SMLO}}$. For the GDR characteristics of the SLO and SMLO models, the recommended values (the energies, widths and weights) from recent database were used (see the Tables 1 and 2 from Ref. [7]). The GDR parameters of the SLO approach were taken for the GLO parameters.

Approximation of a triaxial ellipsoid is used in the TLO model for nuclear shape in deformed nuclei $[14,15]$, and in this approach, the GDR splits into three components and the E1 PSF is described by the expression (3) with $j_{m}=3$ [18, 19]. For the input parameters of the TLO PSF, the resonance energies of the normal modes are taken from the nuclear hydrodynamics model $[14,15,20,21]$ as $E_{r, j}^{\mathrm{TLO}}=E_{0} \cdot R_{0} / R_{j}$, where $E_{0}$ is the resonance energy of the equivalent in volume spherical nucleus with the radius $R_{0}$. The width in the TLO model is independent of gamma-ray energy and the expression with a pow er law dependence on the resonance energy was used $[14,15,22]: \Gamma_{j}^{\mathrm{TLO}}=0.045\left(E_{r, j}^{\mathrm{TLO}}\right)^{1.6}$. The approximation of equally probable excitation of the normal modes of the giant collective vibration were taken for the weights: $s_{j}^{\mathrm{TLO}}=s^{\mathrm{TLO}} / 3$ with $s^{\mathrm{TLO}}=0.995 \simeq 1$ for the sum of the weights.

The triaxial nuclear shape is determined in terms of parameters $\beta$ (represents the extent of quadrupole deformation) and $\gamma$ (the degree of axial asymmetry). Two parameterizations are used here for the calculation of the semi-axis lengths $R_{j}$ for the TLO model. The Hill - Wheeler parameterization [22, 23] is used at experimental values of the deformation parameters $\left(\beta=\beta^{\mathrm{H}}, \gamma=\gamma^{\mathrm{H}}\right)$, which are presented in the Table 1:

$$
R_{j}=R_{j}^{\mathrm{H}}=R_{0} \cdot \exp \left(\sqrt{5 / 4 \pi} \cdot \beta^{\mathrm{H}} \cdot \cos \left(\gamma^{\mathrm{H}}-\frac{2}{3} \pi j\right)\right) .
$$

The Bohr parameterization is taken in [23] with the theoretical deformation parameters $\left(\beta=\beta^{\mathrm{B}}, \gamma=\gamma^{\mathrm{B}}\right)$ :

$$
R_{j}=R_{j}^{\mathrm{B}}=R_{0}\left(1+\sqrt{5 / 4 \pi} \cdot \beta^{\mathrm{B}} \cos \left(\gamma^{\mathrm{B}}-\frac{2}{3} \pi j\right)\right) .
$$

Table 1. The values of deformation parameters $\beta=\beta^{\mathrm{H}}, \gamma=\gamma^{\mathrm{H}}$ used for PSF calculations within TLO(3) model

\begin{tabular}{|c|c|c|c|c|c|c|c|c|c|c|}
\hline Nuclei & ${ }^{94} \mathrm{Mo}$ & ${ }^{98} \mathrm{Mo}$ & ${ }^{146} \mathrm{Nd}$ & ${ }^{148} \mathrm{Sm}$ & ${ }^{156} \mathrm{Gd}$ & ${ }^{168} \mathrm{Er}$ & ${ }^{190} \mathrm{Os}$ & ${ }^{196} \mathrm{Pt}$ & ${ }^{206} \mathrm{~Pb}$ & ${ }^{238} \mathrm{U}$ \\
\hline$\beta$ & -0.08 & 0.18 & 0.17 & 0.13 & 0.22 & 0.28 & 0.16 & 0.13 & 0.02 & 0.29 \\
\hline$\gamma$ & $20^{\circ}$ & $23^{\circ}$ & $26^{\circ}$ & $25^{\circ}$ & $11^{\circ}$ & $12^{\circ}$ & $21^{\circ}$ & $29^{\circ}$ & $40^{\circ}$ & $17^{\circ}$ \\
\hline Ref. & {$[14]$} & {$[14]$} & {$[25]$} & {$[26]$} & {$[27]$} & {$[26]$} & {$[26]$} & {$[14]$} & {$[27]$} & {$[25]$} \\
\hline
\end{tabular}

The deformation parameters in Bohr parametrization (5) were calculated in Ref.[24] for even-even nuclides within framework of the Hartree - Fock -
Bogoliubov theory $\left(\mathrm{HFB} ; \beta^{\mathrm{B}}=\beta_{\mathrm{H}}^{\mathrm{B}}, \gamma^{B}=\gamma_{\mathrm{H}}^{\mathrm{B}}\right)$ and Constrained Hartree - Fock - Bogoliubov approach with five-dimensional collective Hamiltonian 
$\left(\mathrm{CHFB}+5 \mathrm{DCH} ; \beta^{\mathrm{B}}=\beta_{\mathrm{C}}^{\mathrm{B}}, \gamma^{\mathrm{B}}=\gamma_{\mathrm{C}}^{\mathrm{B}}\right)$. In the first order, Bohr's parameters $\left(\beta^{\mathrm{B}}, \gamma^{\mathrm{B}}\right)$ are equal to Hill - Wheeler's parameters $\left(\beta^{\mathrm{H}}, \gamma^{\mathrm{H}}\right)$ [23]. The calculations within TLO model were performed with three variants of the deformation parameters and denoted here as TLO(1), TLO(2) and TLO(3). The corresponding deformation parameters for each model are following: $\operatorname{TLO}(1)-\left(\beta_{\mathrm{H}}^{\mathrm{B}}, \gamma_{\mathrm{H}}^{\mathrm{B}}\right)$; $\operatorname{TLO}(2)-$ $\left(\beta_{\mathrm{C}}^{\mathrm{B}}, \gamma_{\mathrm{C}}^{\mathrm{B}}\right) ; \operatorname{TLO}(3)-\left(\beta^{\mathrm{H}}, \gamma^{\mathrm{H}}\right)$. The deformation parameters within Hill - Wheeler's parameterization for $\mathrm{TLO}(3)$ model were obtained from the fit of experimental data. The Table 1 shows these experimental deformation parameters and corresponding references for 10 isotopes for which the calculations within TLO(3) model were performed.

The comparison of SLO and SMLO approaches for the description of experimental data on photoabsorption cross section was done for 162 nuclei in Ref. [7]. It was shown that the low-energy tails of the photoabsorption cross sections within SLO model are higher than within the SMLO model and higher than experimental data. The comparison between SMLO model and the calculations of E1 PSF within microscopic approaches both quasiparticle randomphase approximation and shell-model was performed in Ref. [13] and demonstrated rather good agreement between them. In this contribution the comparison between SLO and SMLO models with other closedform Lorentzian models of GLO and TLO need to be performed in order to obtain the best description of the experimental photoabsorption cross sections from EXFOR database [28]. Comparing to the Ref. [7], we additionally present the comparisons with the GLO and TLO models. For the TLO model, the atomic nuclei are considered in the triaxial shape approach. In this situation, the corresponding deformation parameters were calculated or obtained for the even-even isotopes only and due to this, we test the E1 PSF for description of the photoabsorption data for the even-even nuclei.

\section{Calculations and discussion}

The analytical PSF expressions for models of SLO, GLO, SMLO and TLO and their input parameters were described in previous section. Quantitative comparison between these PSF models for the description of total photoabsorption cross sections is performed for the following 88 even-even isotopes: ${ }^{24,26} \mathrm{Mg},{ }^{28,30} \mathrm{Si},{ }^{32,34} \mathrm{~S},{ }^{40} \mathrm{Ar},{ }^{40-44,48} \mathrm{Ca},{ }^{46,48} \mathrm{Ti},{ }^{52} \mathrm{Cr}$, ${ }^{54} \mathrm{Fe},{ }^{58,60} \mathrm{Ni},{ }^{64} \mathrm{Zn},{ }^{70,76} \mathrm{Ge},{ }^{76-82} \mathrm{Se},{ }^{88} \mathrm{Sr},{ }^{90,94} \mathrm{Zr}$, ${ }^{92-100} \mathrm{Mo},{ }^{106,108} \mathrm{Pd},{ }^{114} \mathrm{Cd},{ }^{112-124} \mathrm{Sn},{ }^{124-130} \mathrm{Te},{ }^{138} \mathrm{Ba}$ ${ }^{140,142} \mathrm{Ce},{ }^{142-150} \mathrm{Nd},{ }^{144,148-154} \mathrm{Sm},{ }^{152-160} \mathrm{Gd},{ }^{168} \mathrm{Er},{ }^{174} \mathrm{Yb}$, ${ }^{176-180} \mathrm{Hf},{ }^{182-186} \mathrm{~W},{ }^{186-1920} \mathrm{Os},{ }^{194-198} \mathrm{Pt},{ }^{206,208} \mathrm{~Pb},{ }^{232} \mathrm{Th}$, ${ }^{234-238} \mathrm{U}$. The calculations within $\mathrm{TLO}(3)$ model are done for 10 isotopes specified in the Table 1.

For comparison of the theoretical models, the experimental database on photoabsorption cross section was prepared from EXFOR database for eveneven nuclei with the systematic uncertainty less than $10 \%$ (see explanations below). The same datasets were taken as used in Ref. [7] for determination of recommended GDR parameters.

The E1 PSF $\vec{f}^{\alpha}$ from equation (2) is connected with total response of the nucleus on E1 field and defines the total photoabsorption cross section which is given by the relationships:

$$
\begin{gathered}
\sigma(\gamma, \mathrm{abs})=\sigma_{\mathrm{t}}(\gamma, \gamma)+\sigma^{\prime}(\gamma, \mathrm{abs}), \\
\sigma^{\prime}(\gamma, \mathrm{abs})=\sigma(\gamma, \mathrm{sn})+\sigma(\gamma, \mathrm{cp})+\sigma(\gamma, \mathrm{F}), \\
\sigma(\gamma, \mathrm{sn}) \equiv \sigma(\gamma, n)+\sigma(\gamma, 2 n)+\sigma(\gamma, 3 n)+\ldots, \\
\sigma(\gamma, \mathrm{cp}) \equiv \sigma(\gamma, p)+\sigma(\gamma, 2 p)+\ldots+ \\
+\sigma(\gamma, d)+\ldots+\sigma(\gamma, d p)+\ldots+\sigma(\gamma, \alpha)+\ldots .
\end{gathered}
$$

Here, $\sigma_{t}(\gamma, \gamma)=\sigma(\gamma, \gamma)+\sigma\left(\gamma, \gamma^{\prime}\right)$ is total photonscattering cross section with excitation of the nuclear states corresponding to total cross section of target nucleus production, i.e., a sum of the cross sections of elastic gamma-ray scattering via different type of intermediate states (without shape-elastic component like compound-nucleus elastic scattering) and nonelastic gamma-ray scattering; $\sigma^{\prime}(\gamma$, abs $)$ is photoabsorption cross section with emission of the particles together with gamma- rays; $\sigma(\gamma, \mathrm{sn})$ is total photo-neutron reaction cross section; $\sigma(\gamma, \mathrm{cp})$ is photo-charged-particle reaction cross section and $\sigma(\gamma, \mathrm{F})$ is photo-fission reaction cross section for fissionable nuclei, $\sigma(\gamma, \mathrm{F})=\sigma(\gamma, f)+\sigma(\gamma, n f)+$ $+\sigma(\gamma, 2 n f)+\ldots$, with $\sigma(\gamma, f)(\sigma(\gamma, n f))$ for first (second)-chance photofission cross section.

The experimental data for total photoabsorption cross sections from EXFOR database above neutron separation energies $S_{n}$ do not include the contribution of the cross section $\sigma_{t}(\gamma, \gamma)$ from gamma-gamma channels. This contribution is non-negligible at gamma-ray energies $S_{n}+\Delta \varepsilon$ below the threshold of the $(\gamma, 2 n)$ reaction or, in some cases, below the thresholds of other reactions with large cross sections; typically $\Delta \varepsilon<1.5 \mathrm{MeV}$. The absence of this contribution leads to incorrectly small values of $\sigma(\gamma, \mathrm{abs})$ in the gamma-ray range $S_{n}<\varepsilon_{\gamma}<S_{n}+\Delta \varepsilon$.

For every nucleus the specific interval $\Delta \varepsilon$ was found from the condition of ten percent contribution of the cross section $\sigma_{t}(\gamma, \gamma)$ from gamma-gamma 
transitions to the total photoabsorption cross section $\sigma(\gamma, \mathrm{abs})$, i.e. ten percent deviation of $\sigma(\gamma, \mathrm{abs})$ from $\sigma^{\prime}(\gamma$, abs $)$ or ten percent systematic uncertainties of the PSF:

$$
\begin{gathered}
\delta \sigma\left(\varepsilon_{\gamma}=S_{n}+\Delta \varepsilon\right)= \\
=\frac{\sigma_{\mathrm{t}}(\gamma, \gamma)}{\sigma(\gamma, \mathrm{abs})}=\frac{\sigma(\gamma, \mathrm{abs})-\sigma^{\prime}(\gamma, \mathrm{abs})}{\sigma(\gamma, \mathrm{abs})}=0.1 .
\end{gathered}
$$

Cross section, $\mathrm{mb}$

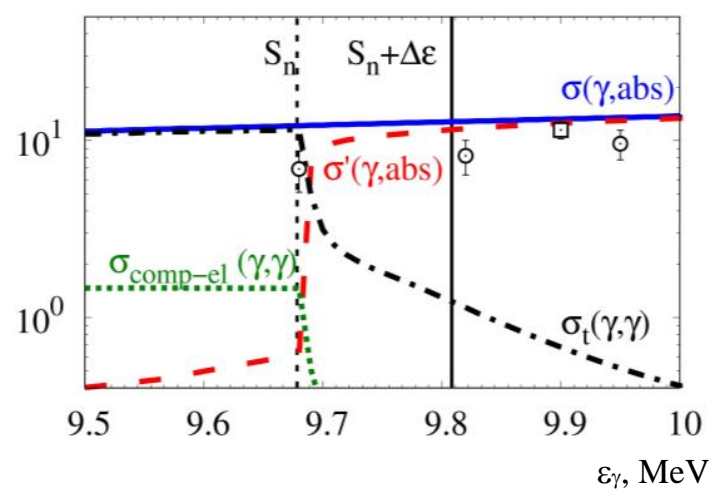

Fig. 1. Theoretically calculated photo cross sections for isotope ${ }^{94} \mathrm{Mo}$ in comparison with experimental data from EXFOR database (circles [29], squares [30]). The vertical lines correspond to $S_{n}$ (black dashed line) and the gamma-ray energy $S_{n}+\Delta \varepsilon$ (black solid line) for which evaluated systematic uncertainties of the PSF are equal to $10 \%$. (See color Figure on the journal website.)

Based on the above, for test of the PSF models for photoabsorption data description, the new database on experimental E1 PSF was prepared using photoabsorption/photo-neutron cross sections from EXFOR database for even-even isotopes with systematic uncertainty less than $10 \%$. In this database the gamma-ray energy interval for each isotope is starting from $\varepsilon_{\min }=S_{n}+\Delta \varepsilon$ and ends in the maximal value of energy $\varepsilon_{\text {max }} \leq 30 \mathrm{MeV}$ presented in the data file. In these intervals above neutron separation energies the gamma-gamma contributions to photo cross sections do not exceed $10 \%$. The specific low boundaries of gamma-ray energy intervals were calculated using simulations of the photo cross sections by the nuclear reaction code TALYS 1.6 [5] with gamma-strength within SLO and others input parameters by default (for example, with Gilbert Cameron model for nuclear level density). It should be noted that in an accordance with TALYS specifications, the cross section $\sigma(\gamma, \mathrm{abs})$ corresponds to the reaction cross section for incident photons $\sigma_{\text {reac }}(\gamma)$, and $\sigma_{t}(\gamma, \gamma)$ is a sum of production cross section $\sigma_{\text {prod }}\left(Z_{\mathrm{t}}, N_{\mathrm{t}}\right)$ of the target nucleus $\left(Z_{\mathrm{t}}, N_{\mathrm{t}}\right)$ and the compound elastic cross section $\sigma_{\text {comp-el }}(\gamma, \gamma): \quad \sigma_{\mathrm{t}}(\gamma, \gamma)=\sigma_{\text {prod }}\left(Z_{\mathrm{t}}, N_{\mathrm{t}}\right)+\sigma_{\text {comp-el }}(\gamma, \gamma)$.
Fig. 1 demonstrates calculated photo-cross sections in comparison with the experimental data for the isotope ${ }^{94} \mathrm{Mo}$.

Two criteria were taken for comparison of the quality of the description of the experimental photonuclear data using different E1 PSF models: 1) minimum of the least-square deviation $\chi_{\alpha}^{2}$, 2) minimum of the root-mean-square (rms) deviation factor $f_{\alpha}[31]$ :

$$
\begin{gathered}
\chi_{\alpha}^{2}=\frac{1}{n} \sum_{i=1}^{n} \frac{\left(\sigma_{\exp }\left(\varepsilon_{i}\right)-\sigma_{\text {the }}^{\alpha}\left(\varepsilon_{i}\right)\right)^{2}}{\left(\Delta \sigma\left(\varepsilon_{i}\right)\right)^{2}}, \\
f_{\alpha}=\exp \left\{\chi_{\mathrm{ln}, \alpha}\right\}, \quad\left(\chi_{\mathrm{ln}, \alpha}\right)^{2}= \\
=\frac{1}{n} \sum_{i=1}^{n}\left\{\ln \sigma_{\text {the }}^{\alpha}\left(\varepsilon_{i}\right)-\ln \sigma_{\exp }\left(\varepsilon_{i}\right)\right\}^{2}=\frac{1}{n} \sum_{i=1}^{n} \ln ^{2}\left(\frac{\sigma_{\text {the }}^{\alpha}\left(\varepsilon_{i}\right)}{\sigma_{\text {exp }}\left(\varepsilon_{i}\right)}\right) .
\end{gathered}
$$

Here $\sigma_{\text {the }}^{\alpha}\left(\varepsilon_{i}\right)=\sigma_{\mathrm{GDR}}^{\alpha}\left(\varepsilon_{i}\right)+\sigma_{\mathrm{qd}}\left(\varepsilon_{i}\right)$ is the theoretical cross section corresponding to Eqs. (1), (2), at gamma-ray energy $\varepsilon_{i} ; \sigma_{\text {exp }}\left(\varepsilon_{i}\right)$ is the experimental photoabsorption cross section from the EXFOR database; $\Delta \sigma\left(\varepsilon_{i}\right)$ is the data uncertainty; $n$ is the number of experimental data points. The rms deviation factor $f_{\alpha}$ corresponds to criterion of a minimum at given gamma-ray energy of the weighted sum of squared deviations of the theoretical cross section from their experimental data in natural logarithmic scale. Because of different estimations of uncertainties in different datasets and, as a rule, for lack of reliable estimations of the systematic errors, a weight of every point is taken as empirical probability $1 / n$. A logarithmic scale is used due to largerange changes of the photoabsorption cross sections.

The $\chi_{\alpha}^{2}$ and $f_{\alpha}$ values were calculated for two gamma-ray energy intervals. The gamma-ray energy intervals from $S_{n}+\Delta \varepsilon$ till $30 \mathrm{MeV}$ (or $\varepsilon_{\max }$ with the energy $\varepsilon_{\max }$ for the last data point) were taken for an estimation of the quality of fitting in the wide energy range and marked below in the tables as "broad range". The short energy ranges near the GDR peaks were taken from the Table 1 of Ref. [7].

For $\chi_{\alpha}^{2}$ calculation, the cross section uncertainties $\Delta \sigma\left(\varepsilon_{i}\right)$ were taken in two versions: 1) the energy-dependent trapezoidal relative uncertainties $\delta \sigma_{\text {trap }}\left(\varepsilon_{i}\right)$ (see below) were applied for all nuclei and marked below as "trap"; 2) the experimental uncertainties $\Delta \sigma_{\text {exp }}\left(\varepsilon_{i}\right)$ given in EXFOR datafiles were used and trapezoidal uncertainty were taken only in case of absence of $\Delta \sigma_{\text {exp }}\left(\varepsilon_{i}\right)$ and marked below as "exp + trap". The energy-dependent trapezoidal rela- 
tive uncertainties $\delta \sigma_{\text {trap }}\left(\varepsilon_{i}\right)=\Delta \sigma_{\text {trap }}\left(\varepsilon_{i}\right) / \sigma_{\text {exp }}\left(\varepsilon_{i}\right)$ were taken in accordance with Ref. [7, 12]. These energy dependent errors were chosen to simulate the statistical error that is inversely proportional to the square root of the counting rate, which has maximum near the GDR. Therefore, the energydependent relative uncertainties were assumed to take minimum values $(10 \%)$ near the GDR peaks and maximum values $(50 \%)$ on the GDR tails. These values are based on the typical uncertainties of the experimental data. For deformed nuclei, a trapezoidal dependence with the GDR peaks as the top corners of the trapezium was assumed as

$\delta \sigma\left(\varepsilon_{i}\right)=\Delta \sigma\left(\varepsilon_{i}\right) / \sigma_{\exp }\left(\varepsilon_{i}\right)=\left\{\begin{array}{l}\delta_{\min }+b\left|E_{r, 1}-\varepsilon_{i}\right|, \quad \varepsilon_{i}<E_{r, 1} \\ \delta_{\min }, \quad E_{r, 2} \leq \varepsilon_{i} \leq E_{r, 1}, \\ \delta_{\min }+b\left|\varepsilon_{i}-E_{r, 2}\right| \quad \varepsilon_{i}>E_{r, 2}\end{array}\right.$

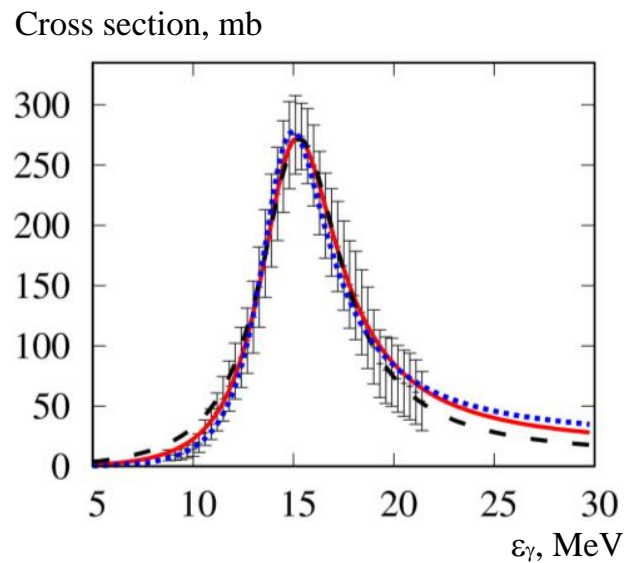

with $b=\left(\delta_{\max }-\delta_{\text {min }}\right) /\left(E_{r, 1}-\varepsilon_{1}\right)$, where $\delta_{\text {min }}=0.1$ and $\delta_{\max }=0.5$ are the minimal and maximal values of uncertainty. For spherical nuclei, a triangular dependence on gamma-energy was used $\delta \sigma\left(\varepsilon_{i}\right)=\delta_{\min }+b\left|E_{r}-\varepsilon_{i}\right|$

Figs. 2 and 3 present the theoretical photoabsorption cross sections calculated within different PSF models in comparison with experimental data for the isotopes of ${ }^{124} \mathrm{Sn}$ and ${ }^{238} \mathrm{U}$. For these isotopes, the relative values of $\chi_{\alpha}^{2} / \chi_{S L O}^{2}$ and $f_{\alpha} / f_{S L O}$ are given in the Table 2 . For the nucleus ${ }^{124} \mathrm{Sn}$, lowest values of $\chi_{\alpha}^{2}$ and $f_{\alpha}$ were obtained by the use of the SMLO and GLO models. For ${ }^{238} \mathrm{U}$, the minimal values of $\chi_{\alpha}^{2}$ and $f_{\alpha}$ correspond to the SMLO and $\mathrm{TLO}(3)$.

Cross section, mb

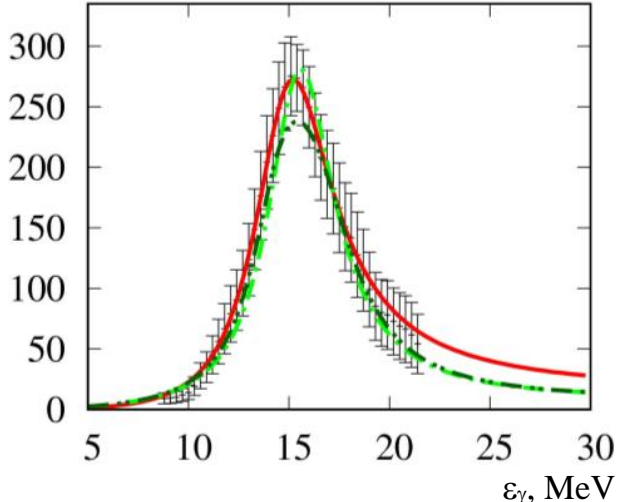

Fig. 2. The experimental cross section data for ${ }^{124} \mathrm{Sn}$ in comparison with calculations within different PSF models. Experimental data are taken from Ref. [32]. Left-hand Figure: - SMLO (red solid line); - - - SLO (black dashed line); •••• GLO (blue dotted line). Right-hand Figure: - SMLO (red solid line); $-\bullet-\bullet-$ TLO(1) (light green dashed followed by one dot line); - - - - TLO(2) (dark green dashed followed by two dots line). (See color Figure on the journal website.)

Cross section, mb

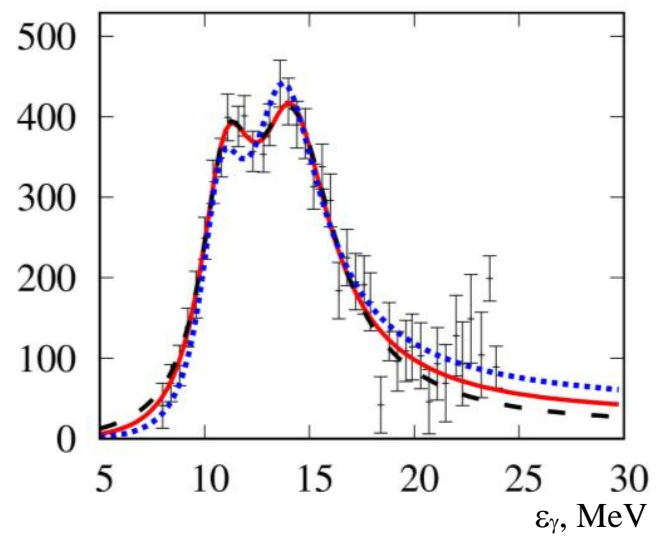

Cross section, $\mathrm{mb}$

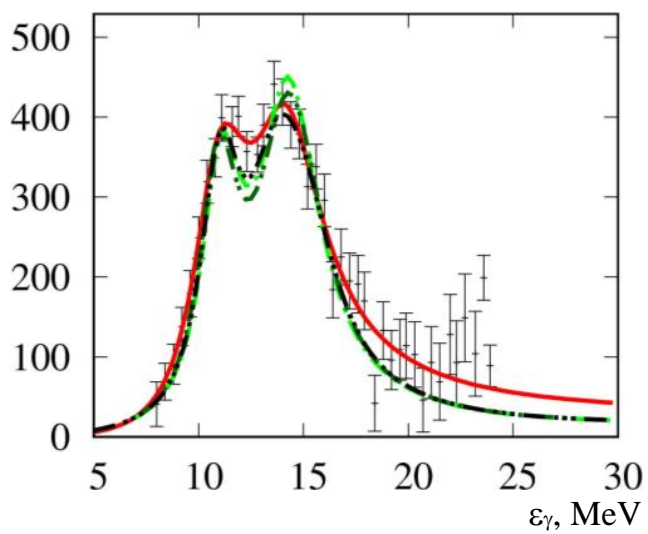

Fig. 3. The experimental cross section data for ${ }^{238} \mathrm{U}$ in comparison with calculations within different PSF models. Experimental data are taken from Ref. [33]. Left-hand Figure: — SMLO (red solid line); - - - SLO (black dashed line), •••• GLO (blue dotted line). Right-hand Figure: — SMLO (red solid line), - •-•- TLO(1) (light green dashed followed by one dot line); - •-•- TLO(2) (dark green dashed followed by two dots line); $-\bullet \bullet-\bullet \bullet-$ TLO(3) (black dashed followed by three dots line). (See color Figure on the journal website.) 
Table 2. The values of $\chi_{\alpha}^{2} / \chi_{\text {SLO }}^{2}$ and $f_{\alpha} / f_{\text {SLO }}$ for ${ }^{124} \mathrm{Sn}$ and ${ }^{238} \mathrm{U}$

\begin{tabular}{|c|c|c|c|c|c|c|c|c|}
\hline Isotope & Criteria & Gamma-ray energy intervals & Uncertainty & SMLO & GLO & TLO(1) & $\mathrm{TLO}(2)$ & $\mathrm{TLO}(3)$ \\
\hline \multirow{4}{*}{${ }^{124} \mathrm{Sn}$} & \multirow{2}{*}{$\chi_{\alpha}^{2} / \chi_{\text {SLO }}^{2}$} & broad range & trap & 0.17 & 0.11 & 0.42 & 0.38 & - \\
\hline & & near GDR & trap & 0.30 & 5.15 & 21.98 & 21.15 & - \\
\hline & \multirow{2}{*}{$f_{\alpha} / f_{S L O}$} & broad range & - & 0.85 & 0.82 & 0.91 & 0.89 & - \\
\hline & & near GDR & - & 0.99 & 1.04 & 1.13 & 1.09 & - \\
\hline \multirow{6}{*}{${ }^{238} \mathrm{U}$} & \multirow{4}{*}{$\chi_{\alpha}^{2} / \chi_{S L O}^{2}$} & \multirow{2}{*}{ broad range } & $\exp$ & 0.81 & 0.97 & 1.83 & 1.90 & 1.55 \\
\hline & & & trap & 1.04 & 1.51 & 1.28 & 1.50 & 1.02 \\
\hline & & \multirow{2}{*}{ near GDR } & $\exp$ & 1.02 & 1.90 & 3.06 & 3.35 & 2.17 \\
\hline & & & trap & 1.15 & 1.63 & 1.34 & 1.64 & 1.03 \\
\hline & \multirow{2}{*}{$f_{\alpha} / f_{S L O}$} & broad range & - & 0.92 & 0.91 & 1.15 & 1.14 & 1.13 \\
\hline & & near GDR & - & 1.01 & 1.04 & 1.03 & 1.02 & 1.02 \\
\hline
\end{tabular}

Table 3. The ratio $K_{\alpha}=<\chi_{\alpha}^{2} / \chi_{\text {SLO }}^{2}>$ for different PSF models

\begin{tabular}{|c|c|c|c|c|c|c|c|}
\hline $\begin{array}{c}\text { Mass numbers } \\
\text { of the isotopes }\end{array}$ & Gamma-ray energy intervals & Uncertainty & SMLO & GLO & TLO(1) & TLO(2) & TLO(3) \\
\hline \multirow{3}{*}{$24 \leq \mathrm{A} \leq 238$} & \multirow{2}{*}{ broad range } & exp + trap & $\mathbf{1 . 1 3}$ & 3.05 & 10.70 & 10.71 & 4.58 \\
\cline { 2 - 7 } & \multirow{2}{*}{ near GDR } & trap & $\mathbf{1 . 0 3}$ & 2.25 & 8.61 & 9.73 & 2.68 \\
\cline { 2 - 7 } & \multirow{3}{*}{ broad range } & exp + trap & $\mathbf{1 . 1 9}$ & 5.43 & 42.98 & 47.88 & 17.28 \\
\cline { 2 - 7 } & trap & $\mathbf{1 . 2 2}$ & 4.86 & 43.07 & 50.08 & 18.17 \\
\hline \multirow{3}{*}{$80 \mathrm{~A} \leq 238$} & exp + trap & $\mathbf{1 . 2 2}$ & 3.58 & 10.94 & 11.33 & 4.58 \\
\cline { 2 - 7 } & \multirow{2}{*}{ near GDR } & trap & $\mathbf{1 . 0 4}$ & 2.54 & 8.28 & 9.58 & 2.68 \\
\cline { 2 - 7 } & & exp + trap & $\mathbf{1 . 2 8}$ & 6.52 & 49.39 & 57.20 & 17.28 \\
\cline { 2 - 7 } & trap & $\mathbf{1 . 2 9}$ & 5.82 & 49.82 & 60.15 & 18.17 \\
\hline
\end{tabular}

Table 4. The ratio $F_{\alpha}=<f_{\alpha} / f_{\text {SLO }}>$ for different PSF models

\begin{tabular}{|c|l|c|c|c|c|c|}
\hline $\begin{array}{c}\text { Mass numbers } \\
\text { of the isotopes }\end{array}$ & $\begin{array}{c}\text { Gamma-ray energy } \\
\text { intervals }\end{array}$ & SMLO & GLO & TLO(1) & TLO(2) & TLO(3) \\
\hline \multirow{2}{*}{$24 \leq \mathrm{A} \leq 238$} & broad range & $\mathbf{0 . 9 7}$ & 1.05 & 1.18 & 1.14 & 1.10 \\
\cline { 2 - 7 } & near GDR & $\mathbf{1 . 0 0}$ & 1.06 & 1.24 & 1.22 & 1.10 \\
\hline \multirow{2}{*}{$80 \leq \mathrm{A} \leq 238$} & broad range & $\mathbf{0 . 9 7}$ & 1.06 & 1.16 & 1.14 & 1.12 \\
\cline { 2 - 7 } & near GDR & $\mathbf{1 . 0 0}$ & 1.05 & 1.20 & 1.18 & 1.10 \\
\hline
\end{tabular}
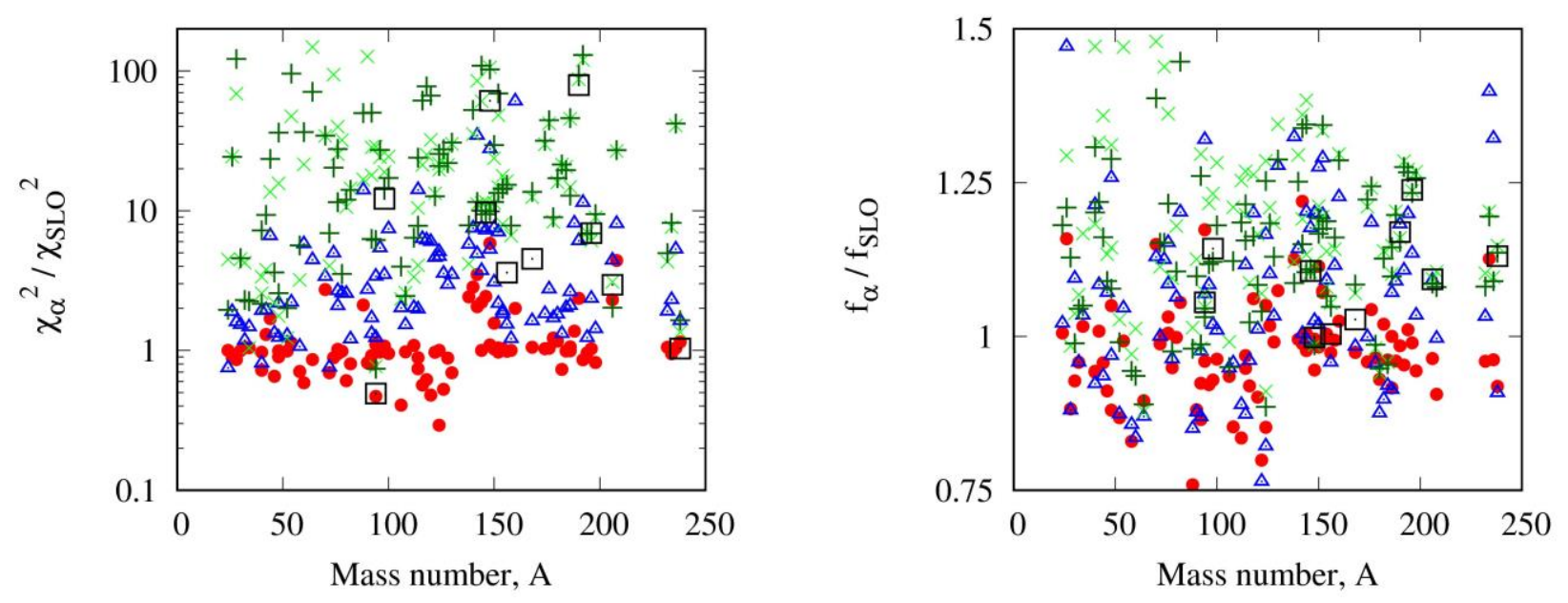

Fig. 4. The relative least square values $\chi_{\alpha}^{2} / \chi_{\text {SLO }}^{2}$ (left-hand Figure) and rms deviation factors $f_{\alpha} / f_{\text {SLO }}$ (right-hand Figure) for even-even isotopes calculated within different PSF models. Presented results correspond to $5-30 \mathrm{MeV}$ gamma-ray energy interval and trapezoidal relative uncertainties. On both Figures: SMLO - red circles ( $\bullet$ ); GLO - blue empty triangles $(\Delta)$; TLO $(1)$ - green crosses $(X)$; TLO $(2)$ - dark green pluses $(+)$; TLO $(3)$ - black empty squares ( $\square$ ). (See color Figure on the journal website.) 
The arithmetic mean values $K_{\alpha}=<\chi_{\alpha}^{2} / \chi_{\mathrm{SLO}}^{2}>$ and $F_{\alpha}=<f_{\alpha} / f_{\text {SLO }}>$ are presented in the Tables 3 and 4 . The relative values of $\chi_{\alpha}^{2} / \chi_{S L O}^{2}$ and $f_{\alpha} / f_{S L O}$ are presented in Fig. 4. On average, the $K_{\alpha}$ and $F_{\alpha}$ have lower values for the SMLO model. The description of the experimental data within the SMLO and GLO models with energy-dependent width is better than within TLO models with standard values of deformation parameters (4), (5).

\section{Conclusions}

Quantitative comparison between the E1 PSF models for the description of total photoabsorption cross sections is performed for 88 even-even isotopes. The database of the photoabsorption cross section was prepared from EXFOR data with systematic uncertainty less than $10 \%$. The uncertainty calculations were performed using TALYS code. The experi- mental values are compared with theoretical predictions within framework of the models SLO, GLO, SMLO and TLO. The criteria of minimum of leastsquare factor and root-mean-square deviation factor were used for comparison of the theoretical calculations with experimental data. It was shown that the SMLO model gives better description of the photoabsorption data in the range of gamma-ray energies till $\sim 30 \mathrm{MeV}$. In our opinion, this model can be recommended in the nuclear reaction codes for adequate modelling of the E1 photoexcitation PSF using simple closed-form expressions.

The authors are very thankful to Tamas Belgya for valuable discussions and comments on TLO model. This work is partially supported by the International Atomic Energy Agency through a Coordinated Research Project on Updating the Photonuclear Data Library and generating a Reference Database for Photon Strength Functions (F41032).

\section{REFERENCES}

1. S. Goriely, M. Wiedeking, P. Dimitriou, Updating the Photonuclear Data Library and Generating a Reference Database for Photon Strength Functions, Summary Report from the First Research Coordination Meeting, INDC(NDS)-0712, 2016.

2. M.B. Chadwick et al. Handbook on Photonuclear Data for Applications: Cross Sections and Spectra. Tech. Rep. IAEA-TECDOC-1178, (IAEA, Vienna, Austria, 2000).

3. R. Capote et al. RIPL - Reference Input Parameter Library for Calculation of Nuclear Reactions and Nuclear Data Evaluations. Nucl. Data Sheets 110 (2009) 3107.

4. M. Herman et al. EMPIRE: Nuclear Reaction Model Code System for Data Evaluation. Nucl. Data Sheets 108 (2007) 2655

5. A.J. Koning, S. Hilaire, M.C. Duijvestijn, TALYS1.0. In: Proc. Intern. Conf. Nuclear Data for Science and Technology (ND2007), Nice, France, April 22 27, 2007. Ed. by O. Bersillon et al. CEA, EDP Sciences, 2008, p. 211.

6. G.A. Bartholomew et al. Gamma-ray strength functions. Adv. Nucl. Phys. 7 (1973) 229.

7. V.A. Plujko et al. Giant Dipole Resonance Parameters of Ground-State Photoabsorption: Experimental Values with Uncertainties. At. Data Nucl. Data Tables 123 - 124 (2018) 1.

8. J. Kopecky, M. Uhl. Test of gamma-ray strength functions in nuclear reaction model calculations. Phys. Rev. C 41 (1990) 1941.

9. V. Plujko et al. Models for photoabsorption cross section estimates. In: Proc. Intern. Conf. on Nuclear Data for Science and Technology (ND2007), Nice, France, April 22 - 27, 2007. Ed. by O. Bersillon et al. CEA, EDP Sciences, 2008, p.235.

10. V. Plujko et al. Verification of models for calculation of E1 radiative strength. Proc. of Science

\section{PoS(PSF07)002.}

11. V.A. Plujko et al. The simplified description of dipole radiative strength function. Int. J. Mod. Phys. E 17 (2008) 240.

12. V.A. Plujko, R. Capote, O.M. Gorbachenko. Giant dipole resonance parameters with uncertainties from photonuclear cross sections. At. Data Nucl. Data Tables 97 (2011) 567.

13. S. Goriely, V. Plujko. Simple empirical E1 and M1 strength functions for practical applications. Phys. Rev. C 99 (2019) 014303.

14. A.R. Junghans et al. Photon data shed new light upon the GDR spreading width in heavy nuclei. Phys. Lett. B 670 (2008) 200.

15. E. Grosse, A.R. Junghans, R. Massarczyk. Breaking of axial symmetry in excited heavy nuclei as identified in giant dipole resonance data. Eur. Phys. J. A 53 (2017) 225.

16. M.B. Chadwick et al. Pauli-blocking in the quasideuteron model of photoabsorption. Phys. Rev. C 44 (1991) 814.

17. T. Belgya et al. Handbook for Calculations of Nuclear Reaction Data, Reference Input Parameter Library-2. Tech. Rep. IAEA-TECDOC-1506 (IAEA, Vienna, Austria, 2006).

18. Y. Alhassid, B. Busch, S. Levit. Thermal Shape Fluctuations, Landau Theory, and Giant Dipole Resonances in Hot Rotating Nuclei. Phys. Rev. Lett. 61 (1988) 1926.

19. Y. Alhassid, B. Busch. Effects of thermal fluctuations on giant dipole resonances in hot rotating nuclei. Nucl. Phys. A 509 (1990) 461.

20. W.D. Myers et al. Droplet model of the giant dipole resonance. Phys. Rev. C 15 (1977) 2032.

21. J.M. Eisenberg, W.M. Greiner. Nuclear Theory. Vol. I: Nuclear Models. 3-rd ed. (North Holland, Amsterdam 1987). 
22. B. Bush, Y. Alhassid. On the width of the giant dipole resonance in deformed nuclei. Nucl. Phys. A 531 (1991) 27.

23. R.W. Hasse, W.D. Myers. Geometrical Relationships of Macroscopic Nuclear Physics (SpringerVerlag, Heidelberg, 1988).

24. J.-P. Delaroche et al. Structure of even-even nuclei using a mapped collective Hamiltonian and the D1S Gogny interaction. Phys. Rev. C 81 (2010) 014303; tables available here.

25. A.R. Junghans et al. A global study of the electric dipole strength in heavy nuclei. Poster 1481 at Intern. Conf. on Nuclear Data for Science and Technology, Island, Jeju, 2010.

26. E. Grosse et al. The energy dependence of the electric dipole strength in heavy nuclei. AIP Conf. Proc. 1090 (2009) 308.

27. E. Grosse et al. Photon strength in spherical and deformed heavy nuclei. EPJ Web of Conf. 8 (2010) 02006.
28. EXFOR, Experimental Nuclear Reaction Data Library.

29. H. Beil et al. A semi-phenomenological description of the giant dipole resonance width. Nucl. Phys. A 219 (1974) 61.

30. H. Utsunomiya et al. Photoneutron cross sections for Mo isotopes: A step toward a unified understanding of $(\gamma, n)$ and $(n, \gamma)$ reactions. Phys. Rev. C 88 (2013) 015805 .

31. A. Gilbert, A.G.W. Cameron. A composite nuclearlevel density formula with shell corrections. Can. Jour. of Phys. 43 (1965) 1446.

32. V.V. Varlamov, M.E. Stepanov, V.V. Chesnokov. New data on photoabsorption reaction cross sections. Bull. Rus. Acad. Sci. Phys. 67 (2003) 724. (Izv. Ross. Akad. Nauk. Ser. Fiz. 67 (2003) 656 (Rus)).

33. G.M. Gurevich et al. Giant resonance in the total photoabsorption cross section of $\mathrm{Z} \approx 90$ nuclei. Nucl. Phys. A 273 (1976) 326.

\author{
В. А. Плюйко ${ }^{1,2}$, С. Горієлі ${ }^{3}$, О. М. Горбаченко ${ }^{1}$, К. М. Солодовник ${ }^{1, *}$ \\ ${ }^{1}$ Київький національний університет імені Тараса Шевченка, Київ, Украӥна \\ ${ }^{2}$ Інститут ядерних досліджень НАН України, Київ, Україна \\ ${ }^{3}$ Інститут астрономї і астрофізики, Університет Брюсселя, Брюссель, Бельгія
}

*Відповідальний автор: e.solodovnyk@gmail.com

\title{
ТЕСТУВАННЯ МОДЕЛЕЙ ФОТОННИХ СИЛОВИХ ФУНКЦЙ ЕЛЕКТРИЧНОГО ДИПОЛЬНОГО ФОТОЗБУДЖЕННЯ
}

Тестуються аналітичні моделі Е1 фотонних силових функцій з опису перерізів фотопоглинання в області енергій гамма-випромінювання 5 - $30 \mathrm{MeB}$ для парно-парних ядер. Було побудовано експериментальну базу перерізів фотопоглинання із систематичною похибкою менше $10 \%$ даних EXFOR. Похибки оцінюються за допомогою теоретичних розрахунків перерізів з використанням коду TALYS 1.6. Теоретичні моделі порівнюються 3 експериментальними даними перерізів фотопоглинання. В якості критеріїв використовуються метод найменших квадратів та середньоквадратичний фактор відхилення. Показано, що проста модифікована модель Лоренца краще описує перерізи при енергіях гамма-випромінювання нижче від 20 МеВ.

Ключові слова: моделі фотонних силових функцій, фотопоглинання, гігантський дипольний резонанс.

\section{В. А. Плюйко ${ }^{1,2}$, С. Гориели ${ }^{3}$ А. Н. Горбаченко ${ }^{1}$ Е. Н. Солодовник ${ }^{1, *}$ \\ ${ }^{1}$ Киевский национальный университет имени Тараса Шевченко, Киев, Украина ${ }^{2}$ Институт ядерных исследований НАН Украины, Киев, Украина \\ ${ }^{3}$ Институт астрономии и астрофизики, Университет Брюсселя, Бельгия \\ *Ответственный автор: e.solodovnyk@gmail.com \\ ТЕСТИРОВАНИЕ МОДЕЛЕЙ ФОТОННЫХ СИЛОВЫХ ФУНКЦИЙ ЭЛЕКТРИЧЕСКОГО ДИПОЛЬНОГО ФОТОВОЗБУЖДЕНИЯ}

Тестируются аналитические модели Е1 фотонных силовых функций для описания сечений фотопоглощения в области энергий гамма-излучения 5 - 30 МэВ для четно-четных ядер. Подготовлена экспериментальная база сечений фотопоглощения с систематической погрешностью менее $10 \%$ из данных EXFOR. Погрешности оцениваются при помощи теоретических расчетов сечений с использованием кода TALYS 1.6. Теоретические модели сравниваются с экспериментальными данными сечений фотопоглощения. В качестве критериев используются метод наименьших квадратов и среднеквадратический фактор отклонения. Показано, что простая модифицированная модель Лоренца лучше описывает сечения фотопоглощения при энергиях гамма-излучения ниже $\sim 30 \mathrm{MэB.}$

Ключевые слова: модели фотонных силовых функций, фотопоглощение, гигантский дипольный резонанс. 